Result. The results of this project demonstrated a total reduction in hypnotic tablet administration was very significant with a $44.5 \%$ reduction post intervention.

Conclusion. This demonstrates the positive change in our clinical practice that has resulted from our interventions. This will improve patient safety and reduce cost of hypnotic medications for the NHS. Following on from this initial intervention, we feel that we can continue to make further changes and expand the changes we made on this ward, to other similar wards in our hospital, trust and to other inpatient psychiatric wards further afield.

\section{A qualitative study of foundation year two (F2) doctor's attitudes towards psychiatry carried out in Northern Ireland}

Michael Doris $^{1 \star}$, Kathyrn Mitchell ${ }^{1}$, Damien Hughes ${ }^{1}$, Lorraine Parks ${ }^{2}$ and Angela Carragher ${ }^{2}$

${ }^{1}$ Belfast HSC Trust and ${ }^{2}$ South Eastern Health and Social Care Trust ${ }^{*}$ Corresponding author.

doi: 10.1192/bjo.2021.111

Aims. Recruitment into psychiatry is a major issue nationally. Northern Ireland (NI) consistently punching above it's weight with psychiatry recruitment - in a region that only attracts $31.8 \%$ of F2s to enter into any training programme, Core psychiatry has been consistently oversubscribed. Here we look to examine the experiences of F2s in NI, including those who have had a placement in psychiatry and those who have not - what can we learn from NI? Background. The exposure to psychiatry during the F2 year is a crucial time for recruitment to psychiatry. In NI, where there has been an $100 \%$ fill rate at core training level for many years, trainees and consultants have pointed towards a positive experience in the F2 year.

Method. Questionnaires were given out at a sample of F2 Generic Skills sessions, gathering a range of quantitative and qualitative data. A representative sample of over half of current F2s wrote about there preconceptions and experiences of psychiatry, whether they had worked in it or not. An a priori approach was taken towards generating codes as part of a framework analysis from which 4 major themes were identified.

Result. 93/148 F2 doctors who were approached responded to the survey of which $36.6 \%$ had experienced a Foundation placement in psychiatry. Major qualitative themes that emerged were exposure to psychiatry, the nature of working in psychiatry, being valued and stigma. Doctors who had an F2 placement were much more likely to be willing to pursue a career in it, regardless of whether they had been allocated a placement with psychiatry by choice or not.

Conclusion. This survey adds to the literature that exposure to psychiatry in undergraduate and postgraduate level has a huge role in shaping attitudes towards the specialty of psychiatry, and indeed the likelihood of a foundation doctor going on to become a psychiatry trainee. Stigma in the medical profession towards mental illness and psychiatry remains prevalent.

\section{Benefits observed in an inpatient MDT programme} for FND are not associated with medication use or previous therapies

\author{
Thomas Elliott ${ }^{1 \star}$ and Michael Elmalem ${ }^{2}$ \\ ${ }^{1}$ Camden and Islington NHS Foundation Trust and ${ }^{2}$ National \\ Hospital for Neurology and Neurosurgery \\ ${ }^{\star}$ Corresponding author.
}

doi: 10.1192/bjo.2021.112
Aims. The National Hospital for Neurology and Neurosurgery provides various services for patients with Functional Neurological Disorder (FND), including a four-week inpatient rehabilitation programme run by an integrated Multi-Disciplinary Team (MDT) of Occupational Therapists (OT), Physiotherapists (PT), Psychologists and Psychiatrists.

We had observed that patients with FND often have medical and psychiatric comorbidities including affective, dissociative, somatic symptom and pain disorders; pharmacological treatments are commonly used. We hypothesised that a high burden of medication, particularly of those which produce dependence, might limit one's ability to entrain therapeutic strategies and therefore benefit from treatment. We additionally hypothesised that patients who had previously tried individual physical or psychological therapies might gain less than those who were treatment-naïve.

Method. In this service evaluation project, we reviewed records from 97 consecutive elective inpatient admissions, comprising the entire intake for 2019 and 2020. Data were extracted from the inpatient discharge summary and therapies discharge report of each patient. We recorded which therapies for FND patients had previously tried (OT; PT; Speech and Language Therapy; Psychology; Pain Service) and the classes of medications they were taking on admission (opiates; benzodiazepines; antidepressants; mood stabilisers; antipsychotics; gabapentinoids). We compared the differentials in outcome measures recorded on the first and last day, including the Canadian Occupational Performance Measure $(n=79)$ and EQ-5D-5L $(n=79)$. Statistical tests of effect size and significance were done using SPSS-25. Group comparisons of EQ-5D-5L were made with Paired t-tests; all other comparisons were done with Wilcoxon signed-rank tests due to non-normal data.

Result. The most common medications used were antidepressants (72\%), gabapentinoids (39\%), opiates (36\%) and benzodiazepines (25\%). $69 \%$ of patients had tried PT, $57 \%$ psychology and $52 \%$ OT, while only $13 \%$ were treatment naïve. Whole-cohort analysis revealed highly significant improvements $(\mathrm{p}<0.001)$ in occupational performance, satisfaction, ratings of general health, subjective difficulty in performing tasks and in pain and fatigue levels. We found no significant differences in outcome measures that correlated with past therapies or medication use.

Conclusion. Our analysis shows that the great majority of our patients gained meaningful benefits from their admission, both on clinician-rated metrics of occupational performance and patient-rated measures of subjective improvement. That there was no significant relationship with therapies or medications suggests, promisingly, that patients taking various medications and with suboptimal responses to previous therapy can still benefit from our MDT programme. Limitations include correlational design, limited generalisability to the general population, missing data for certain outcome measures and the absence of follow-up data.

To resuscitate or not to resuscitate; a question for old age psychiatrists

Liam Embliss* and Mohan Bhat

Goodmayes Hospital, North East London NHS Foundation Trust ${ }^{*}$ Corresponding author.

doi: 10.1192/bjo.2021.113

Aims. The inpatient population of an older adult psychiatric ward will include people with physical and mental health conditions which affect life span and quality of life. Patients may be frail, acutely unwell, or have terminal illnesses such as dementia. It is 